\title{
El uso de smartphones y tablets en Educación Infantil. Una propuesta de investigación que empodera a la infancia ${ }^{1}$
}

\author{
Ana Castro-Zubizarreta $\left({ }^{*}\right)$, María Carmen Caldeiro-Pedreira $\left({ }^{* *}\right)$ y María M. Rodríguez-Rosell $\left({ }^{* *}\right)$ \\ ${ }^{(*)}$ Universidad de Santiago de Compostela - España $\left(^{* *}\right)$ Universidad de Murcia - España (**) Universidad Católica de Murcia - España
}

\section{RESUMEN}

El ecosistema mediático actual y la omnipresencia de pantallas se han convertido en un elemento protagónico en la sociedad hiperconectada. Cada vez a edades más tempranas los menores, denominados como nativos digitales, están en contacto con la tecnología. Hoy en día contamos con numerosos estudios que confirman el contacto directo con dispositivos electrónicos y tecnología móvil, sin embargo, existe una marcada carencia de investigaciones que den voz a la infancia y que muestren su óptica e intereses.

Ante esta necesidad y teniendo en cuenta el derecho de la infancia a participar y a ser escuchada, se ha diseñado una herramienta que permita visibilizar sus intereses y darles voz. El instrumento está elaborado según tres dimensiones: provisión, protección y participación que se estructuran entorno a diferentes categorías que justifican el valor de las diferentes técnicas de recogida de información que componen el instrumento. La herramienta se utilizará en ambientes adecuados para la participación buscando espacios conocidos para los niños, cómodos y en los que no se sientan intimidados ya que el instrumento se pasará a los niños por parejas o por tríos para mitigar, en la medida de lo posible, la disparidad de poder del adulto respecto a los pequeños.

Palabras Clave: smatphone, tablet, visibilización, infancia, empoderamiento.

\section{The use of smartphones and tablets in childhood education. A research proposal to empower children}

\section{ABSTRACT}

The current media ecosystem and the omnipresence of screens have become leading elements into hyperconnected society. Minors have been appointed as digital natives and they are in touch with technology at youngest ages. Today we have a lot of studies that confirm real contact with electronic devices and mobile technology, however there is a remarked lack of researches that attributes voice to children and it allow them to show their views and their interests.

Because of this, we need take into account the right of children to participate and the right to be heard. In this way we have designed a tool that allows us to show their interests; a tool which allows us to give voice to children. The instrument has been built according to three dimensions: provision, protection and participation which are designed around different categories. These categories justify the value of different techniques which are made up the instrument. In this way, we used it to obtain information. We will use this tool in appropriate environments for participation; we will be looking for familiar spaces for children, comfortable environments in which they do not feel intimidated. The instrument will be applied to children in little groups; we will use this technique in order to delete adult's influence, as far as possible.

Keywords: smatphone, tablet, visibility, childhood, empowerment.

\section{La digitalización de la infancia}

El nuevo ecosistema mediático (Canavilhas, 2011) y la interactividad son dos elementos protagónicos en la actualidad. Hoy en día los nuevos medios entre los que situamos no solo a los ordenadores sino además los móviles o las tablets, se erigen como una fuerza dominante importante en la sociedad en general y entre el colectivo de la infancia, en particular. Cada vez más se reduce la edad en la que se establece un contacto directo con los dispositivos electrónicos y la tecnología móvil. Un hecho que se produce no solo en España y Europa sino además en el continente latinoamericano donde hace más de un lustro los niños

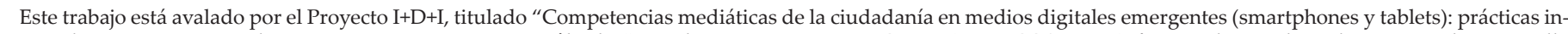

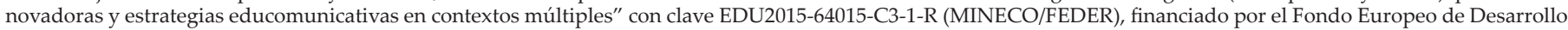
Regional (FEDER) y Ministerio de Economía y Competitividad de España.
} 
en edad infantil se convertían en protagonistas del nuevo sector de consumo de teléfonos móviles (Duet, Enriz, Tourn y Muñoz, 2012).

En este sentido, y posibilitado por el inminente contacto con la tecnología, en el entramado mediático más reciente existen vocablos que resultan familiares a la mayoría de las personas; entre otros, sociedad red, sociedad multipantalla, tecnologización del conocimiento o adolescentes conectados (Vidales-Bolaños y Sádaba-Chalezquer, 2017) son algunos de los términos que pueblan el contexto cotidiano. Independientemente del poder adquisitivo o del lugar geográfico en el que nos situemos, refiriéndonos en este caso a España, apreciaremos el contacto de casi cualquier persona de 0 años con la tecnología. Dada la preocupación que conlleva el uso de estas herramientas a estas edades, han surgido informes como el dirigido por García-Galera (2008); investigaciones que desde hace una década focalizan la atención en el uso, influencias y responsabilidad de la telefonía móvil en la infancia y adolescencia.

Un contacto que trataremos de analizar, en esta ocasión, en relación con la infancia. En este caso, conviene matizar que se concibe con carácter amplio como el momento que se extiende desde que el niño nace hasta la etapa de Educación Primaria, un período en el cual la familia, las tecnologías o el entorno son algunos de los agentes formativos determinantes. Unos y otros condicionan el desarrollo del menor, especialmente durante los primeros años de vida y contribuyen a su formación. Por tanto, en el diseño de investigación que presentamos, más allá de la visión restringida de la Educación Infantil que se circunscribe a la etapa del sistema educativo de 0-6 años, nos referimos de manera amplia a la educación que se lleva a cabo en la familia o fuera de ella, durante el periodo inicial de la vida del niño y que comprende desde el nacimiento hasta su ingreso en la escolaridad obligatoria. Hace referencia, por tanto, al conjunto de intervenciones o acontecimientos educativos familiares, ambientales, escolares..., intencionales o no que recibe el niño durante este periodo de su vida.

Conscientes de la enorme influencia que los móviles y las tablets ejercen sobre el colectivo infantil y teniendo en cuenta que la convivencia con ellos se produce a edades cada vez más tempranas, se ha considerado la necesidad de conocer cuál es la percepción de los niños sobre la provisión (acceso, uso, tiempos y entornos), protección (supervisión y control parental) y participación (preferencias, ocio e intereses) sobre estos dispositivos.

Consideramos que en una sociedad tecnificada como la actual el uso precoz del móvil y la Tablet se instaura como habitual. Una premisa que certifica Roca (2015) en el Informe FAROS del Observatorio de Salud de la Infancia y la Adolescencia, dependiente del Hospital Sant Joan de Déu de Esplugues. En este estudio se afirma que un $40 \%$ de la población menor de dos años utiliza Tablets y móviles. Además de esto, señala también que, tanto los niños como las niñas comienzan de forma prematura, desde bebés, a utilizar la tecnología. Una idea que se perfilaba hace años cuando se ha acuñado el término "nativo digital" (Prensky, 2001, 2005; White y Le Cornu, 2011) que ha suscitado tanta controversia a lo largo de la última década.

Este vocablo, más allá de caracterizar a los usuarios de estos dispositivos que conforme avanza el tiempo van incrementando las horas de uso, nos sirve para centrar el interés de la presente investigación. Nuestro estudio busca dar voz a los niños y niñas, usuarios nativos de los dispositivos móviles. Pretende conocer desde su propio punto de vista la presencia que tiene el móvil en sus vidas reconociendo el valor de sus palabras y el sentido y significado que confieren a las experiencias que obtienen a través del mismo. Apuntamos así sobre la necesidad de abordar un reto para la investigación educativa, el reconocer la participación infantil en la investigación, dar valor a sus aportaciones y superar así el sesgo de las investigaciones que únicamente recogen la perspectiva del adulto sobre temas que experimentan los niños y de los que son los principales protagonistas.

Por tanto, en este artículo presentaremos el diseño y validación de un instrumento que ha sido creado para que los niños más pequeños puedan expresarse y participar a través de él en la investigación. Un instrumento que pretende recoger y respetar a la vez que dar valor a los lenguajes infantiles, recogiendo su imaginario de primera mano, sin la mediación de los adultos, otorgando a los niños un rol protagónico en el proceso investigador. Además de ello se pretende contar con la información necesaria para no solo dar voz a la infancia sino además empoderarla ante los potenciales riesgos de la Red y formarla, desde un inicio, para utilizar de manera adecuada las herramientas tecnológicas que a priori y debido a su corta edad utiliza y/o consume de forma intuitiva e insuficientemente segura, tal y como recomienda UNICEF (2014), en su informe sobre derechos de la infancia en la sociedad digital. En el contexto digital más inmediato resulta fundamental el alcance no solo de prosumidores (García-Ruiz, Ramírez-García y Rodríguez-Rosell, 2014) sino de prodiseñadores activos (Hernández, Renés, Graham y Greenwill, 2017), es decir, personas que desde las edades más tempranas han de desarrollar la capacidad que les permita convivir entre los contenidos audiovisuales. Una necesidad que justifica el hecho de que tanto las pantallas como las diferentes tecnologías y dispositivos móviles no son únicamente herramientas comunicativas fundamentales, sino que se erigen como elementos estructurales básicos que contribuyen a la formación de los adultos y también de los más pequeños. (De-Casas-Moreno, Caldeiro-Pedreira y Rodríguez-Romero, 2018).

El contexto y condiciones señaladas certifican la valía del estudio que presentamos y justifican su necesidad en un momento en el cual los padres y madres de familia acostumbran a estar absortos ante el ordenador o inmersos en responder correos mientras sus descendientes necesariamente deben entretenerse de formas no siempre propias de su edad; en ocasiones los dispositivos tecnológicos se convierten por un momento en "niñera digital" (García-Rodríguez y Gómez-Díaz, 2016); es decir elementos que los menores utilizan de manera inadecuada en su tiempo de ocio, mientras los mayores atienden a su trabajo o simplemente se comunican a través de las tecnologías más recientes. El WhatsApp o diferentes Apps móviles sirven para que los menores ocupen su tiempo de forma impropia a sus edades (ONTSI, 2014).

En este sentido, diferentes estudios atribuyen aspectos como la mala alimentación de los menores a un uso desproporcionado de la tecnología por parte de los progenitores o la falta de atención de los mismos. Una falta que se presume al excesivo grado de atención a las herramientas tecnológicas (Pediathrics, 2014) y que se contrapone a la afirmación que hace referencia a la mayor flexibilidad laboral que permiten la digitalización o la interactividad, un hecho que podría considerarse, a priori y desde esta óptica como un factor con un marcado carácter positivo a la hora de valorar su potencialidad.

\section{Supervisión y control como formas de proteger y empoderar a la infancia del siglo XXI}

La infancia del s-XXI a la que nos hemos referido requiere de un guía que supervise y aconseje sin limitar ni prohibir el uso de los dispositivos móviles. Tal premisa resulta fundamental en un momento en el cual la edad de contacto con la tecnología, según 
se ha constatado, se ha reducido notablemente. En esta situación y dado el contagio existente entre mayores y menores a la hora de acceder a la Red y de utilizar los diferentes dispositivos resulta deseable por parte de los adultos el alcance de la autonomía mediática (Caldeiro, 2014) y la competencia crítica (Caldeiro y Aguaded, 2015) que convergen en la adecuada comunicación e interrelación de la ciudadanía mediática (Gozálvez, 2013) que puebla la sociedad hiperconectada más reciente.

En este entorno sería deseable el control de los adultos sobre el uso de las herramientas tecnológicas por parte de los menores, no solo los de 12 años quienes, según indica el Estudio sobre hábitos seguros en el uso de Smartphones por los niños y adolescentes españoles, ya disponen de móvil, sino también de aquellos que, ocasionalmente lo utilizan. Una necesidad que se ha detectado desde finales de la primera década del actual siglo cuando ha sido publicado el Estudio sobre hábitos seguros en el uso de las TIC por niños y adolescentes y e-confianza de sus padres (Inteco, 2009) en el cual se constatan algunos de los riesgos a los que se enfrentaban los menores en el uso de las Tic, entre los que destacan la vulneración de derechos de propiedad industrial o intelectual, el acceso a contenidos inapropiados, la interacción $\mathrm{y}$ acecho por otras personas y/o ciberbullying, el grooming y/o acoso sexual o las amenazas a la privacidad. Aspectos que, a lo largo de los años han ido alcanzando un mayor protagonismo llegando a focalizar la atención de titulares y noticias de carácter negativo que reflejan la realidad social más reciente. Esta situación y el hecho de que cada vez se produzca a edades más tempranas ha justificado la publicación de trabajos en los cuales "se aborda el impacto y efectos de nuevas pantallas digitales como la tablet en el desarrollo infantil" (Nogueira y Ceinos, 2015:33). En esta línea contamos con diferentes guías para padres y educadores sobre el uso seguro en Internet, móviles y videojuegos (Labrador, Requens y Helguera, 2015); asimismo destaca la proliferación de volúmenes que centran la atención en la seguridad en las Redes (Flores, 2009) o la protección online de la infancia y la adolescencia (Flores, 2011). En esta línea conviene citar la Web Internet Segura for kids https://goo.gl/GDUcmG, un espacio que contiene diferentes recursos de Control Parental que, según figura en la propia Web "permite a los padres mantenerse informados sobre la ubicación de sus hijos, así como administrar el tiempo de uso del dispositivo, bloquear aplicaciones que no les parezcan adecuadas, conocer quiénes son sus contactos".

Se trata de materiales enfocados a los adultos que deben asesorar a jóvenes que superan los 10-12 años. Sin embargo, se ha detectado una marcada carencia de recursos enfocados al colectivo infantil de edades tempranas (inferiores a los 6 años), y que utiliza los móviles y las Tablets de forma ocasional y sin visibilizar. Una situación, que urge no solo advertir, sino que es necesario contar con los recursos y materiales necesarios para enfrentarse a ella.

Por otra parte, existe una cierta disparidad entre los niños y niñas de menos de 10-12 años y los que superan esas edades, dado que no solo se constata la inexistencia de materiales y la falta de conciencia de la situación, sino que se aprecia una invisibilización y silenciamiento de este colectivo. Factor este último que justifica el interés de esta investigación pues se ha abordado en la literatura reciente el efecto y repercusiones del uso de Smartphone y Tablets en el aprendizaje infantil (Ron, Álvarez y Núñez, 2013), el impacto del uso del teléfono móvil en las relaciones interpersonales de los niños (Martínez, Encino y Gonzá-

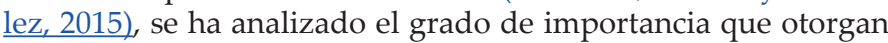
los niños a los dispositivos móviles en su vida (Duek et al., 2012) y el consumo de dispositivos digitales por niños y adolescentes (Contreras-Espinosa, García y González, 2015) entre otros focos temáticos que, comparten entre sí el hecho de que si se cuenta en ellos con la participación de los niños ésta se contempla en los últimos cursos de Educación Primaria y Educación Secundaria, siendo la etapa de Educación Infantil y por lo tanto los niños más pequeños, los ausentes en las investigaciones desarrolladas hasta el momento. Rescatar del silencio la perspectiva de los pequeños confiere al diseño de investigación que presentamos en este artículo un valor añadido, dada su originalidad, relevancia y pertinencia. Además de ello se considera fundamental el papel de la educación a la que se ha hecho referencia al inicio de este trabajo. Esta, no puede permanecer ajena a la situación mediática actual dado que, «es en el ámbito de la educación de los hijos donde el peligro de exponer a los consumidores a contenidos que no están basados en evidencias, que presentan valores sesgados, entornos de aprendizaje digital pobres y con fines comerciales ocultos es presumiblemente mayor que en contextos formales de educación» (Suárez-Perdomo, Byrne y Rodrigo, 2018:21).

\section{Superar el adultocentrismo en la investigación educativa: la mirada infantil en la investigación como tendencia emergente}

La Convención de los Derechos del Niño (1989) supuso reconocer el derecho de la infancia a ser tenida en cuenta, a que ésta sea reconocida como un colectivo con derecho a participar y a ser escuchada en aquellos temas que la afectan. La participación infantil se erige como uno de los principios fundamentales de la citada Convención que reivindica la creación de espacios de convivencia e interacción donde la voz de los niños no sea silenciada sino valorada, considerando sus aportaciones como valiosas, parte de una forma particular de entender el mundo y de construir significados. Al respecto, la investigación educativa requiere responder al reto de generar investigaciones donde tenga cabida la participación de una infancia protagónica, donde se presente a los niños y niñas como actores sociales, ciudadanos activos, con derechos, competentes y expertos en sus propias vidas, no meros ejecutores dependientes de un adulto (Pascal y Bertram, 2009; Harcourt y Einarsdottir, 2011). Estos planteamientos abren la necesidad de superar el sesgo de muchos estudios que recogen la perspectiva adulta sobre la temática de estudio que priman la visión adultocentrista que se instaura como hegemónica en la tradición de la investigación educativa. Disminuir este sesgo y contemplar la perspectiva infantil favorecerá la creación de un conocimiento más profundo y amplio de la temática de estudio, pero también un conocimiento mayor de la propia infancia, que se descubre y presenta al mundo a través de su participación.

En la última década el interés por recoger el punto de vista de los niños sobre temas que afectan a su vida se ha convertido en un área de interés para los investigadores sobre todo en el contexto escolar, donde los niños y sus aportaciones son recogidas como vías esenciales para la construcción de escuelas más democráticas y participativas y como mecanismos de mejora de la propia escuela. Algunos estudios son los abordados por Fielding, (2011) y Messieu, (2013), sin embargo, los niños que se encuentran en la etapa de Educación Infantil son los menos escuchados siendo escasos los estudios que recogen su participación en la investigación (James y Prout, 1990; Castro y Manzanares, 2015).

Si dentro del contexto escolar la participación de la infancia en la investigación educativa la entendemos en ciernes y se presenta como una vía a seguir en otras áreas de investigación, fuera del contexto escolar podemos decir que este desafío se incrementa al encontrarnos ante un terreno casi inexplorado. De ahí nuestro interés por recoger el punto de vista que los niños tienen sobre el uso del Smartphone en su vida, la supervisión que tienen al respecto, sus preferencias de uso en la vida cotidiana y 
en sus momentos de ocio y las aplicaciones y posibilidades que los niños perciben de este dispositivo. De esta forma consideramos que con esta investigación posibilitaremos uno de los derechos de la infancia recogidos en la Convención y potenciaremos el reconocimiento de la infancia y de su capacidad de actuación dentro de los escenarios sociales, lo que no significa que los niños que participarán en el estudio tengan la verdad absoluta, a modo de infantocracia, sino que pretendemos conocer el estado de la cuestión a estudiar reconociendo el valor de sus aportaciones, que serán contrastadas con las aportaciones adultas, de familias y profesorado, lo que nos permitirá tener un conocimiento global del fenómeno de estudio, triangulando perspectivas y promoviendo la reflexión y el diálogo de todos los informantes.

\section{Algunas cuestiones clave en el diseño de la investigación con niños sobre dispositivos móviles (Smartphone)}

\subsection{La búsqueda de técnicas que promuevan la participación} de los niños

La participación de los niños en la investigación educativa supone encontrar aquellas técnicas que faciliten su plena participación, la recogida de sus opiniones, sentimientos y pareceres. Se presenta por tanto como un requisito indispensable para poder lograr una investigación con niños exitosa que posibilite recoger los múltiples lenguajes de la infancia, cargados de sentido y significado y cuyo valor se pierde cuando la técnica de recogida de información utilizada los obvia o limita su escucha. Por ello, hemos considerado fundamental acercarnos al conocimiento de las denominadas "técnicas amigas de los niños" ampliamente descritas en trabajos de Castro, Argos y Ezquerra (2011; 2016) que comparten las siguientes características: son conocidas por los niños, son de su gusto y agrado y fáciles de desarrollar. Entre ellas podemos señalar el uso del dibujo (Einarsdottir, 2007;
Harris y Barne, 2009); la realización de fotografías (Coleyshaw, Whitmarsh, Jopling, y Hadfield, 2012); las conversaciones con niños (Theobald, y Kultti, 2012) o el enfoque mosaico entre otras (Clark y Moss,2001). Técnicas que entendemos no cerradas a las anteriormente nombradas sino en constante construcción y dependientes de la creatividad y capacidad de escucha del investigador.

\subsection{El diseño del instrumento y el proceso de validación del mismo}

\subsubsection{Las dimensiones del instrumento}

El diseño del instrumento se inicia con la delimitación de las dimensiones de estudio. Para ello, el estudio se inspira en el análisis de los tres ejes fundamentales de la Convención de los Derechos del niño, conocidos como las tres P: Provisión, Protección y Participación (Rodríguez et al., 2012) y que adoptamos como grandes dimensiones de análisis que orientarán la investigación: a) provisión, entendido como acceso de los niños al uso de esta tecnología móvil; b) protección, que en nuestro caso hace referencia a las condiciones de riesgo para la protección infantil y las garantías de supervisión y apoyo de las que los niños se sientan partícipes y c) participación, en el sentido de reconocer las preferencia infantiles sobre el uso de dichos dispositivos en su vida cotidiana y su presencia y toma de decisiones sobre las actividades de ocio en su tiempo libre, además de entender la participación de los niños en la investigación como informantes de primer orden en las dimensiones presentadas. Estas dimensiones y sus categorías acotadas en este diseño se presentan en la siguiente figura, si bien, consideramos que en el análisis de los resultados puedan identificarse otras categorías que identificaremos como emergentes y que se incluirán en el análisis de los resultados.

Figura 1. Dimensiones y categorías que componen el instrumento

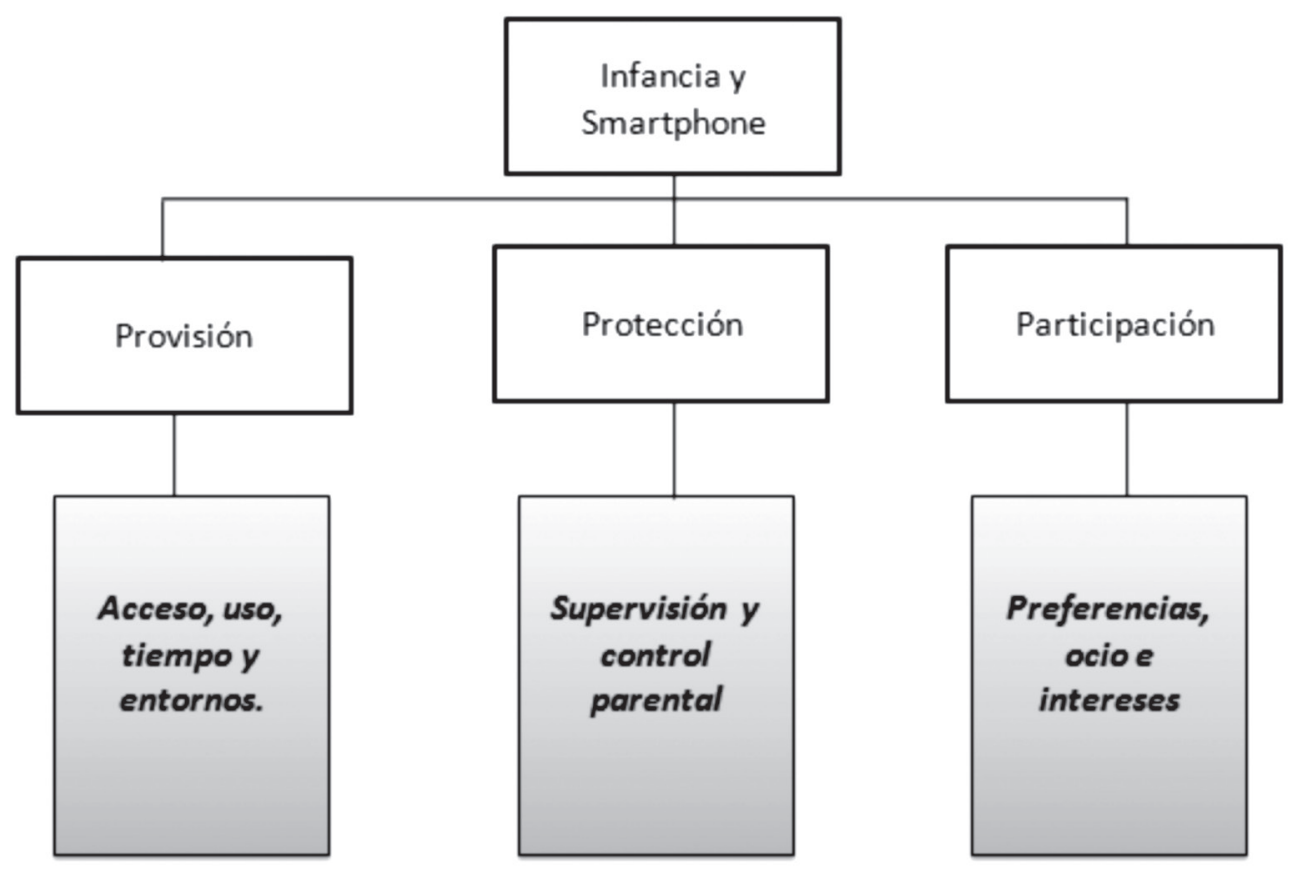


4.2.2. Las técnicas de recogida de información que componen el instrumento

- Conversaciones con niños. Esta técnica requiere de la presentación de interrogantes a los pequeños sobre el contenido que se pretende conocer, para lo que es necesario la construcción de un espacio donde los niños se sientan libres de poder hablar y expresar sus opiniones e ideas para lo que en las conversaciones los niños participarán por parejas o por tríos. Siguiendo las orientaciones de Mayall (2000) se debe dejar el protagonismo a los niños en las conversaciones, y a través de los interrogantes planteados se ha de permitir que se esta- blezcan diálogos entre los niños y las aportaciones individuales han de enriquecerse con las contribuciones sucesivas de los otros niños. Se trata de una técnica desarrollada ya desde los años 80 por Mayall (2000) y que ha sido desarrollada por investigadores tanto de ámbito internacional (Borzone y Rosemberg, 2000; Dockett y Perry, 2005; Marguetts, 2008; Theobald, y Kultti, 2012;) y nacionales (Castro y Manzanares, 2015; Castro, Ezquerra y Argos, 2016). A través de ella se busca la recogida de información sobre las dimensiones provisión y protección. Siguiendo estas indicaciones se presenta la siguiente tabla que muestra posibles interrogantes para la recogida de información.

Tabla 1. Guión conversaciones con niños

$\begin{array}{ll}\text { DIMENSIÓN } & \text { CATEGORÍAS } \\ \text { PROVISIÓN } & \text { Acceso } \\ & \text { Uso } \\ & \text { Entornos }\end{array}$

Tiempos

PROTECCIÓN Supervisión y control parental

\section{POSIBLES INTERROGANTES}

¿Qué es esto? ¿Para qué sirve? ¿Te dejan el móvil? ¿Tú lo usas? ¿Te gusta usarlo? ¿Qué haces con el móvil? Si juegas... ¿a qué juegas? ¿Haces fotografías con él? ¿Haces vídeos con él? ¿Ves fotografías o vídeos que hay en el móvil? ¿Crees que es para que lo usen los mayores o para niños? ¿Dónde usas el móvil? ¿En qué sitios? (si no contestan en la escuela, reforzar para conocer datos al respecto) ¿Quién te deja el móvil?

¿En qué momento puedes estar con el móvil? ¿Te dejan el móvil todos los días o algunos días? ¿Puedes usar el móvil todo el tiempo que tú quieras? Cuando te dejan el móvil antes de empezar a usarlo, ¿te dicen hasta cuándo te lo dejan? ¿Crees que usas el móvil mucho tiempo? ¿Te gustaría usar el móvil más tiempo del que haces?

¿Tienes que pedir permiso para coger el móvil? ¿Puedes coger el móvil cuando tú quieras? ¿Dónde está papá y mamá cuando tú estás con el móvil? Si estás con otra persona, por ejemplo, tu hermano o hermana, utilizando el móvil a la vez, ¿quién decide lo que veis? ¿Puedes ver en el móvil todo lo que tú quieras o es papá o mamá quien te dice lo que puedes ver o no? ¿Hay juegos o programas descargados en el móvil que te gustan? ¿Te dejan descargar los juegos/videos de Youtube que tú quieras? ¿Pides permiso para descargar un juego y ver vídeos de Youtube? ¿Prefieres usar el móvil solo o con ayuda de los mayores?
- El dibujo. La técnica del dibujo ha sido ampliamente utilizada en la evaluación psicopedagógica. Bien conocidas son las técnicas del dibujo del árbol o de la familia, sin embargo, en nuestro estudio se utilizará como medio de expresión de los niños al presentarse como una técnica familiar del gusto y agrado de los más pequeños que recogerá otra forma de comunicación o de expresión de estos, sobre todo como medio de inclusión en la investigación de quienes no tengan unas habilidades lingüísticas muy desarrolladas pudiendo así visibilizar sus ideas a los demás y ser de esta forma tenidos en cuenta. Muestra del uso de esta técnica utilizada bajo en enfoque de la participación en la investigación son los estudios desarrollados por Einarsdottir, (2007), Harris y Barne (2009); Dockett y Perry (2005). En la técnica del dibujo se solicitará a los niños que en un folio dividido en dos por la mitad dibujen en un lado qué es lo que hacen con el móvil en su tiempo libre y en el otro lado de la hoja dibujen qué es lo que les gustaría hacer en su tiempo libre.

- El juego con tarjetas de imágenes. Inspirándonos en Einarsdottir (2005), optamos por la presentación de tarjetas con imágenes para que los pequeños nos indiquen sus preferencias y la presencia del smartphone en su vida cotidiana y en su tiempo de ocio. Se presentan seis tarjetas con fotografías de diferentes actividades y se les dará la siguiente consigna: De todas estas actividades dime cuál sería la actividad que elegirías. ¿y después de esa, cuál otra? ¿Por qué has elegido esa? ¿Con quién te gustaría estar? Hay algo que te gustaría hacer que no aparece, ¿qué? El juego con tarjetas de imágenes brinda al niño la posibilidad de manipularlas, de ordenarlas según sus preferencias y de plantear los por qué de sus elecciones, así como la posibilidad de recoger comentarios espontáneos sobre actividades o elementos que no se hayan contemplado.

\subsubsection{La validación del instrumento}

La validez del contenido del instrumento creado se llevó a cabo a través de dos procedimientos. En primer lugar, se optó por la evaluación mediante el juicio de expertos, entendido como "una opinión informada de personas con trayectoria en el tema, que son reconocidas por otros como expertos cualificados en éste, y que pueden dar información, evidencia, juicios y valoraciones" (Escobar-Pérez y Cuervo-Martínez, 2008: 28). Al respecto, Cabero y Llorente (2013) advierten que la correcta selección de los jueces y su nivel de experticia será determinante. Por ello, con el propósito de conocer si las preguntas y técnicas planteadas en el instrumento eran adecuadas y relevantes para recoger información sobre las dimensiones objeto de estudio se realizó una consulta a 6 jueces expertos que fueron seleccionados atendiendo a dos criterios: el dominio del ámbito de las tecnologías de la información y la comunicación, concretamente dispositivos móviles y, por otro lado, a su vinculación docente con la Educación Infantil. De esta forma tres de los expertos fueron profesores doctores universitarios cuya línea de investigación se vincula 
con la tecnología educativa y la educación mediática y otros tres fueron maestras de Educación Infantil.

A los evaluadores expertos se les aportó una planilla de evaluación siguiendo el modelo propuesto por Escobar-Pérez y Cuervo-Martínez (2008) que recogía los siguientes indicadores: suficiencia, claridad, coherencia y relevancia. A continuación, se recogen las aportaciones más significativas que los jueces expertos trasladaron en sus valoraciones y fueron recogidas por el equipo investigador para mejorar el instrumento. Una constante que aparece en todas las aportaciones es la necesidad de que el contenido del instrumento resulte claro y fácil de comprender para los niños. Las aportaciones de las jueces expertas en Educación Infantil ayudaron a adecuar el lenguaje a la población infantil. En este sentido éstas son algunas de sus aportaciones:

- Los niños en Educación Infantil todavía no tienen bien adquirida la noción del tiempo, las preguntas referidas a esta categoría tienen que reformularse en torno a si a los niños los adultos les permiten estar todo el tiempo que quieren con el móvil, si les dicen hasta cuándo, por ejemplo, hasta la merienda, o si quieren estar más tiempo con él.

- Para conocer la supervisión o el control parental del uso del móvil por parte de los niños es necesario concretar más la pregunta, por ejemplo, ¿dónde están papá y mamá mientras tú estás jugando con el móvil?

- Uno de los jueces apunta también la necesidad de que una vez los niños finalicen el dibujo se les pida que verbalicen lo plasmado en el papel y esto sea apuntado por las investigadoras.

Las valoraciones que los jueces efectuaron del instrumento fueron muy positivas animando a implementar el instrumento una vez se tuvieron en cuenta sus aportaciones y se realizaron las modificaciones sugeridas. El segundo paso realizado para validar el instrumento consistió en su aplicación a una pequeña muestra de cuatro niños comprobando la viabilidad del mismo, tanto en tiempo de aplicación como en su claridad y gusto de los pequeños por las actividades que se les pedían realizar. En este sentido, contar con la participación de los niños para validar el instrumento es una evidencia más de cómo a través de esta investigación se pretende dar voz a los niños y otorgarles un papel activo en la misma.

\section{Consideraciones éticas a tener en cuenta para la implemen- tación del estudio}

Una investigación que pretenda contar con población infantil como informante de primer orden requiere tener muy presente la ética que ha de orientar e inspirar el desarrollo del estudio. Para ello, en primer lugar, se ha considerado fundamental que la investigación sea aprobada por la Comisión de Ética de las Universidades a las que están adscritas las investigadoras. En segundo lugar, se ha reflexionado sobre el papel o rol que ejercerán los niños en la investigación, destacando sus derechos y capacidades durante todo el proceso investigador. Para ello, siguiendo las pautas aportadas por Ortiz, Prats y Baylina (2012) se ha primado el consentimiento informado como vía no solo de suministrar información clara del objeto de estudio a las personas adultas sino también a los propios niños, explicándoles por qué entendemos importante su participación y pidiéndoles su consentimiento para participar o no en el estudio, esto es, la decisión final la tiene el niño y no el adulto. Se garantizará que todas las opiniones serán recogidas, entendiéndolas como importantes, así como su anonimato.

Los principios morales que, por tanto, orientarán la investigación serán los propuestos por Graham, et al., (2013) quienes señalan los siguientes: respeto, beneficio y justicia. El respeto como reconocimiento de la infancia, de su dignidad y capacidad que se ve reflejado en el consentimiento informado. El beneficio entendido en primer lugar como la inclusión de todos los niños y que ésta no plantee ningún daño para los pequeños. En segundo lugar, como la necesidad de promover que la investigación tenga una repercusión positiva en la vida de los niños. Finalmente, la justicia que se plantea como la necesidad de disminuir el poder del adulto ante el niño, dando el mismo valor a sus aportaciones que a aquellas que son realizadas por adultos.

\section{Aspectos conclusivos: Una propuesta de investigación que empodera la infancia}

Los niños conviven en un contexto mediático protagonizado por un "imparable desarrollo de las tecnologías de la información y la comunicación" (Ramírez-García, Renés-Arellano y Aguaded, 2015) un contexto en el cual impera la presencia de dispositivos móviles y pantallas que está omnipresente de forma incuestionable en sus vidas. El acceso cada vez más temprano por parte de la infancia a los mismos requiere de un estudio profundo que nos ayude a comprender mejor la vida de los niños en la actualidad lo que posibilitará un mayor conocimiento de la infancia. Para ello, el diseño de investigación presentado anteriormente asegura la participación de los niños en el estudio y que sus experiencias sean tenidas en cuenta cumpliendo con uno de los planteamientos recogidos en la Declaración de los Derechos del Niño (1989).

La investigación en la que participan los niños es de vital importancia pues, tal y como refieren Graham et al., (2013) proporcionará una información precisa y culturalmente específica de gran valor que garantiza la validez de los resultados. En esta línea entendemos que con esta propuesta de investigación se tendrán presentes las tres grandes condiciones que según Trilla y Novella (2001) exhiben la participación real y auténtica de los niños: reconocer que tienen derecho a participar, tener la capacidad para ejercer dicho derecho adaptando las técnicas de recogida de información y proveer de escenarios y ambientes adecuados para la participación, lo que se hará buscando espacios conocidos para los niños, cómodos y en los que no se sientan intimidados ya que el instrumento se pasará a los niños por parejas o por tríos para mitigar, en la medida de lo posible, la disparidad de poder del adulto respecto a los pequeños.

Con todo, entendemos que este tipo de investigaciones son de gran interés para la comunidad educativa, pero coincidimos con Novella (2012:382) cuando afirma que "todavía nos queda mucho camino por recorrer, aunque tengamos las bases para hacerlo nos falta aquello más esencial que es la disposición a hacerla real y posible".

\section{Referencias bibliográficas}

Borzone, A., y Rosemberg, C. (2000). ¿Qué aprenden los niños cuando aprenden a hablar? El desarrollo lingüístico y cognitivo en los primeros años. Buenos Aires: Aique.

Cabero, J. y Llorente, M.C. (2013). La aplicación del juicio de experto como técnica de evaluación de las tecnologías de la información (TIC). Eduweb. Revista de Tecnología de Información y Comunicación en Educación, 7 (2) pp.11-22.

Caldeiro, M.C., y Aguaded-Gómez, J. (2015). «Estoy aprendiendo, no me molestes» la competencia mediática como forma de expresión crítica de nativos e inmigrantes digitales. Redes.com, 12. (http://goo.gl/2ORkf1)

Caldeiro-Pedreira, M.C. (2014) Alfabetización comunicativa para el desarrollo de la autonomía mediática. Estudio de la com- 
petencia mediática de los adolescentes de Lugo (Galicia). (http:// goo.gl/1UJ4Nm)

Canavilhas, J. (2011). El nuevo ecosistema mediático. Index. comunicación, 1(1), 13-24. Recuperado de https://goo.gl/RLYKwP

Castro, A., y Manzanares, N. (2015). Los más pequeños toman la palabra: la Escuela Infantil que a ellos les gustaría, Revista Complutense de Educación, 27(3), 923-941.

Castro, A.; Ezquerra, P., y Argos J. (2011). Dando voz y protagonismo a la infancia en los procesos de investigación e innovación educativos, Revista Fuentes, 11, 107-123.

Castro, A.; Ezquerra, P., y Argos J. (2016). Procesos de escucha y participación de los niños en el marco de la educación infantil: Una revisión de la investigación. Educación XX1, 19(2), 105-126.

Clark, A. y Moss, P. (2001). Listening to Young Children: The Mosaic Approach. London: National Children's Bureau.

Coleyshaw, L.; Whitmarsh, J.; Jopling, M., y Hadfield, M. (2012). Listening to children's perspectives: improving the quality of provision in early years settings. Part of the Longitudinal Study of Early Years Professional Status. Research Report DFE-RR239b, University of Wolverhampton.

Contreras, Espinosa, RS., García Medican, I., \& González, Z. (2015). Consumo de medios digitales por niños y preadolescentes en Cataluña, España. ZER, 20(39), 145-162.

De Casas-Moreno, P.; Caldeiro-Pedreira, M.C., y Rodríguez-Romero, L.M. (2018). La televisión como espejo de la realidad del «bullying». Percepciones de los adolescentes sobre el programa «Proyecto Bullying». Aula Abierta, 47(2), pp. 193-202. DOI: https://doi.org/10.17811/rifie.47.2.2018.

Dockett, S., y Perry, B. (2005). Starting school in Australia is a "bit safer, a lot easier and more relaxing": Issues for parents from culturally and linguistically diverse backgrounds. Early Years, 25(3), 271 - 281.

Duek, C.; Enriz, N.; Tourn, G., y Muñoz, F. (2012). Niños, teléfonos móviles y consumo: nuevas prácticas con nuevas tecnologías. Infancia Imágenes, 11(1), 9-17.

Einarsdottir, J. (2005). We can decide what to play! Children's perception of quality in an Icelandic playschool. Early Education and Development, 16(4), 469-488.

Einarsdottir, J. (2007). Research with children: Methodological and ethical challenges. European Early Childhood Education Research Journal, 15(2), 197-211.

Escobar-Pérez, J. y Cuervo-Martínez, A. (2008). Validez de contenido y juicio de expertos: una aproximación a su utilización. Avances en medición, 6(1), 27-36.

Fielding, M. (2011). La voz del alumnado y la inclusión educativa: una aproximación democrática radical para el aprendizaje intergeneracional. Revista Interuniversitaria de Formación del Profesorado, 70(25,1), 31-61.

Flores, J. (2011). Diez claves para la protección online de la infancia y la adolescencia. Recuperado de https://goo.gl/JCdZU1

Flores, J. (2009). Seguridad en las Redes Sociales. Recuperado de https://goo.gl/4x3SsZ

García-Galera, M.C. (Dir.) (2008). La telefonía móvil en la infancia y adolescencia. Usos, influencias y responsabilidades. Madrid: oficina del defensor del menor en la Comunidad de Madrid.

García-Rodríguez, A., y Gómez-Díaz, R. (2016) Lectura digital infantil. Dispositivos, aplicaciones y contenidos; Barcelona: UOC.

García-Ruiz, R., Ramírez, A., y Rodríguez, M.M. (2014). Media Literacy Education for a New Prosumer Citizenship. [Educación en alfabetización mediática para una nueva ciudadanía prosumidora]. Comunicar, 43, 15-23. https://doi.org/10.3916/C432014-01
Gozálvez, V. (2013). La ciudadanía mediática. Una mirada educativa. Madrid:Dyckinson.

Graham, A., Powell, M., Taylor, N., Anderson, D., y Fitzgerald, R. (2013). Ethical Research Involving Children. Florence: UNICEF Office of Research - Innocenti.

Harcourt, D., y Einarsdóttir, J. (2011). Introducing children's perspectives and participation in research, European Early Childhood Education Research Journal, 19(3), 301-307.

Harris, K., y Barnes, S. (2009). Male teacher, female teacher: Exploring children's perspectives of teachers' roles in kindergartens. Early Child Development and Care, 179(2), 167-181.

INTECO (2009). Estudio sobre hábitos seguros en el uso de las TIC por niños y adolescentes y e-confianza de sus padres. Observatorio de Seguridad de la Información. Recuperado de https://goo. $\mathrm{gl} / 1 \mathrm{~T} 8 \mathrm{YCb}$

James, A., y Prout, A. (1990). Constructing and Reconstructing Childhood. Contemporary Issues in the Sociological Study of Childhood. London: Falmer Press.

Labrador, F.; Requesens, A., y Helguera, M. (2015). Guía para padres y educadores sobre el uso seguro de Internet, móviles y videojuegos. Madrid: Fundación Gaudium. Recuperado de https://goo. $\mathrm{gl} / \mathrm{jUaFSO}$

Margetts, K. (2008). Transition to school: What children think about how it works and how it is going to be different things. Conferencia presentada en EECERA, Stavanger

Mayall, B. (2000). Conversations with children: working with generational issues. En P. Christensen, \& A. James, Advocating for children: International Perspectives on Children's Rights (págs. 120135). London: Falmer.

Martínez, M., Enciso, R., y González, S. (2015). Impacto del uso de la tecnología móvil en el comportamiento de los niños en las relaciones interpersonales. Educateconciencia, 5(6), 67-80.

Messiou, K. (2013). El compromiso con la voz del alumnado: uso de un marco de trabajo para abordar la marginación en las escuelas. Revista de Investigación en Educación, 11(3), 97-108.

Nogueira, MA \& Ceinos, C. (2015). Influencia de la tablet en el desarrollo infantil: perspectivas y recomendaciones a tener en cuenta en la orientación familiar. Tendencias pedagógicas, 26, pp. 33-50. Recuperado de https://goo.gl/MN8DH6

Novella, A.M. (2012). La participación infantil: Concepto dimensional en pro de la autonomía ciudadana. Revista Teoría de la Educación: Educación y Cultura en la Sociedad de la Información, 13(2), 380-403.

Organización de las Naciones Unidas (ONU) (1989). Convención de los Derechos del Niño. [formato html]. http://www. un.org/es/events/childrenday/ pdf/derechos.pdf (Consultado el 9 de octubre de 2013).

Ortiz, A., Prats, M., y Baylina, M. (2012). Métodos visuales y geografías de la infancia: dibujando el entorno cotidiano. Scripta Nova. Revista Electrónica de Geografía y Ciencias Sociales. [En línea], XVI(400).

Pascal, C. y Bertram, T. (2009). Listening to young citizens: the struggle to make real a participatory paradigm in research with young children, European Early Childhood Education Research Journal, 17, (2), 249-262.

Pediathrics (2014). Patterns of Mobile Device Use by Caregivers and Children During Meals in Fast Food Restaurants. Recuperado de DOI: 10.1542/peds.2013-3703

Prensky, M. (2005). Teaching digital natives: partnering for real learning. Recuperado de https://goo.gl/opELD9

Prensky, M. (2001). Digital Natives, Digital Immigrants. New York: MCB University Press.

Ramírez-García, A.; Renés-Arellano, P. y Aguaded, I. (2016). La competencia mediática en los criterios de evaluación del cu- 
rrículo de Educación Primaria. Aula Abierta 44, pp. 55-62. http:// dx.doi.org/10.1016/j.aula.2015.08.002 0210-2773/

Roca, G. (Coord.) (2015) Las nuevas tecnologías en niños y adolescentes. Guía para educar saludablemente en una sociedad digital. Barcelona: Hospital Sant Joan de Déu. Recuperado de http:// faros.hsjdbcn.org

Rodríguez, I., Gualda, E., Barrero, N., Arjona, A., Checa, J.C y Rodríguez, A. (2012). La población infantil ante las nuevas tecnologías de la información. Una aproximación a la realidad de los nativos digitales andaluces. Colección Actualidad. Fundación Pública Andaluza Centro de Estudios Andaluces, Consejería de la Presidencia, Junta de Andalucía.

Ron, A. Álvarez y P. Núñez (2013). Los efectos del marketing digital en niños y jóvenes: Smartphones y tablets ¿enseñan o distraen?, España: ESIC Editorial.

Suárez-Perdomo, A., Byrne, S. y Rodrigo, M. (2018). Assessing the ethical and content quality of online parenting re- sources. [Evaluación de la calidad ética y del contenido de los recursos online para padres]. Comunicar, 54, 19-28. https://doi. org/10.3916/C54-2018-02

Theobald, M. A. y Kultti, A. (2012). Investigating child participation in the everyday talk of teacher and children in a preparatory year. Contemporary Issues in Early Childhood, 13(3), 210-225.

Trilla, J. y Novella, A. (2001). Educación y participación social en la infancia. Revista Iberoamericana de Educación, 26, 137-164.

Vidales-Bolaños, M. y Sádaba-Chalezquer, C. (2017). Connected Teens: Measuring the Impact of Mobile Phones on Social Relationships through Social Capital. Comunicar, 53, 19-28. https:// doi.org/10.3916/C53-2017-02

Web Internet Segura for kids https://goo.gl/GDUcmG

White, D. y Le Cornu, A. (2011). Visitors and Residents: A new typology for online engagement. First Monday, 9(16). Recuperado de http://dx.doi.org/10.5210/fm.v16i9.3171 